\title{
Comparison and Evaluation of Mitotic Figures in Oral Epithelial Dysplasia using Crystal Violet and Feulgen Stain
}

\author{
${ }^{1}$ Roopa S Rao, ${ }^{2}$ Shankargouda Patil, ${ }^{3}$ Anveeta Agarwal
}

\section{ABSTRACT}

Background: Routine staining procedures often pose a problem in differentiating a mitotic cell from an apoptotic cell, deteriorating the reliability of histology grading. Although various new methods have been recommended for identifying mitotic figures (MFs) in tissues, the time factor and cost makes them less feasible. Thus, an attempt was made to evaluate the efficacy of crystal violet and Feulgen reaction in identifying MFs and also to see for any variation in the number of MFs in various grades of Epithelial dysplasia.

\section{Objectives:}

1. Using crystal violet and Feulgen stain in the identification and counting of MFs on diagnosed cases of epithelial dysplasia and thereby to evaluate their efficacy.

2. To evaluate the variation in the number of MFs in various grades of epithelial dysplasia.

Materials and methods: The study sample includes retrieval of 30 formalin fixed paraffin embedded tissue sections diagnosed for different grades of epithelial dysplasia (WHO grading system, 2005) from the archives, Department of Oral Pathology, MSRDC, Bengaluru. Ten tissue sections each of mild, moderate and severe epithelial dysplasia were stained with $\mathrm{H} \& \mathrm{E}$, Feulgen and $1 \%$ crystal violet stains and the number of MFs were counted. Five cases of cervical carcinoma were taken as control. Stained sections were compared, and data obtained was statistically analyzed using the Kruskal-Wallis test.

Results: A significant increase in the number of MFs ( $p=$ 0.02 ) was observed in Feulgen stained sections as compared to H\&E stain.

Conclusion: Feulgen stain can be considered as a simple, reliable, cost-effective and reproducible method of staining MFs.

\footnotetext{
${ }^{1}$ Professor and Head, ${ }^{2}$ Associate Professor

${ }^{3}$ Postgraduate Student

1,3 Department of Oral Pathology, MS Ramaiah Dental College Bengaluru, Karnataka, India

${ }^{2}$ Department of Oral Pathology and Microbiology, Faculty of Dental Sciences, MS Ramaiah University of Applied Sciences Bengaluru, Karnataka, India

Corresponding Author: Shankargouda Patil, Associate Professor, Department of Oral Pathology and Microbiology Faculty of Dental Sciences, MS Ramaiah University of Applied Sciences, Bengaluru-560013, Karnataka, India, e-mail: sbpatil1612@gmail.com
}

Keywords: Mitotic figures, H\&E stain, Feulgen stain, $1 \%$ crystal violet stain, Oral epithelial dysplasia.

How to cite this article: Rao RS, Patil S, Agarwal A. Comparison and Evaluation of Mitotic Figures in Oral Epithelial Dysplasia using Crystal Violet and Feulgen Stain. J Contemp Dent Pract 2014;15(3):273-277.

\section{Source of support: Nil}

Conflict of interest: None declared

\section{INTRODUCTION}

Mitosis is a process of nuclear division where the replicated DNA molecules of each chromosome are divided into two nuclei accompanied by cytokinesis leading to formation of two identical daughter cells. Mitosis serves as the basis of producing new cells and has not only a role in basic cell biology but also a breakdown in the cell's ability to regulate its own division leading to major implications. ${ }^{1}$

Mitotic figures (MFs) refer to the various phases of mitosis namely prophase, metaphase, anaphase and telophase, which are appreciated in tissue sections. ${ }^{2}$ Increased and atypical number of MFs has been included as a criteria in the WHO classification for grading oral epithelial dysplasia (1978). These MFs are a valuable tool in assessing cellular proliferation and act as prognostic indicators in oral epithelial dysplasia.

Routinely, hematonylin and cosin (H\&E) stain is used for estimation of MFs. Although advanced methods, like IHC, flow cytometry, autoradiography, DNA ploidy, are available for the identification of MF, but their increased cost and time makes them difficult to use; hence, special stains can be employed for rapid and easy identification. Toluidine blue, giemsa, crystal violet, have been used in the past to stain the nuclear DNA. ${ }^{2,3}$

Feulgen and crystal violet are special stains used in histology to identify chromosomal material in cell specimens. These are dependable on acid hydrolysis of DNA. The present study is an attempt to aid in the distinct and differential staining of MFs which will facilitate easy identification using Feulgen and $1 \%$ crystal violet stains, also to assess and evaluate the number of MFs in various grades of oral epithelial dysplasia. 


\section{MATERIALS AND METHODS}

The study sample included retrieval of 30 formalin fixed paraffin embedded tissue sections diagnosed for various grades of oral epithelial dysplasia from archives of Department of Oral Pathology, MSRDC, Bengaluru.

Study samples were grouped into two categories: group 1 comprised of control group of cervical carcinoma $(n=5)$ cases and group 2 consisted of 30 diagnosed cases of oral epithelial dysplasia $(n=30)$ graded based on WHO grading system (2005) proposed by Warnakulasuriya et al. ${ }^{4} \mathrm{Ten}$ cases each of mild, moderate and severe epithelial dysplasia were taken.

\section{Methodology}

Three serial sections of 5 microns each were sectioned and stained with $1 \%$ crystal violet, Feulgen stain and H\&E stain using the methodology from Godkar et al and Bancroft respectively. ${ }^{5,6}$ Sections were interpreted for MFs under $4 \times$, $10 \times$ and $40 \times$ magnification, under a binocular microscope Olympus CX21. The criteria given by Van Diest et al were strictly adopted to identify MFs in order to rule out similar looking cells like a pyknotic nuclei, apoptotic cell, etc.

Table 1: Mitotic figures in three grades of epithelial dysplasia using three staining methods

\begin{tabular}{llll}
\hline & H\&E stain & $\begin{array}{l}1 \% \text { crystal } \\
\text { violet stain }\end{array}$ & $\begin{array}{l}\text { Feulgen } \\
\text { stain }\end{array}$ \\
\hline $\begin{array}{l}\text { Mild epithelial } \\
\text { dysplasia }(\mathrm{n}=10)\end{array}$ & 1.05 & 1.65 & 2.35 \\
$\begin{array}{l}\text { Moderate epithelial } \\
\text { dysplasia }(\mathrm{n}=10)\end{array}$ & 5.3 & 6.35 & 7.5 \\
$\begin{array}{l}\text { Severe epithelial } \\
\text { dysplasia }(\mathrm{n}=10)\end{array}$ & 12.0 & 15.15 & 17 \\
\hline
\end{tabular}

Table 2: $p$-values observed

\begin{tabular}{ll}
\hline Stains & $p$-value \\
\hline H\&E and crystal violet & 0.324 \\
H\&E and Feulgen & 0.021 \\
Crystal violet and Feulgen & 0.078 \\
\hline
\end{tabular}

Van Diest et als criteria to assess the MFs are as follows:

1. The nuclear membrane must be absent indicating the cells have passed the prophase.

2. Clear, hairy extension of nuclear material (condensed chromosome) must be present, either clotted (beginning metaphase) in a plane (metaphase/anaphase) or in separate clots (telophase).

3. Two parallel, clearly separate chromosomes clot to be counted individually as if they are separate mitosis.

The entire stretch of the epithelium was observed and the number of MFs in each field was counted in a step ladder fashion and summed up as shown in Figure 1, to give the total number of MFs in that particular tissue section.

Areas excluded for counting MFs are as follows:

1. Areas showing necrosis

2. Inflammation

3. Tissue folds and calcifications.

\section{Statistical Methods}

The entire procedure was blinded using three observers. Data was statistically analyzed using Kruskal-Wallis ANOVA test. Interobserver variability was calculated using Kappa statistics.

\section{RESULTS}

A total of 30 cases of epithelial dysplasia were retrieved of which 10 each were mild, moderate and severe epithelial dysplasias. The number of MFs was compared among the three groups, it was seen that a significant increase in the number of MFs was observed in sections stained with Feulgen stain as compared to $1 \%$ crystal violet and H\&E stains (Table 1 ). A significant $(p<0.01)$ increase in the identification of MFs was observed in Feulgen stained sections as compared to the gold standard H\&E.

We checked for any variation in the number of MFs in different grades of epithelial dysplasia, but no significant increase in the number of MFs between mild, moderate

Table 3: Average number of MFs counted by the two observers under 10x

\begin{tabular}{|c|c|c|c|c|c|c|c|c|c|}
\hline \multirow[t]{2}{*}{ S. no. } & \multicolumn{3}{|c|}{ Mild epithelial dysplasia } & \multicolumn{3}{|c|}{ Moderate epithelial dysplasia } & \multicolumn{3}{|c|}{ Severe epithelial dysplasia } \\
\hline & $H \& E$ & $C V$ & Feulgen & $H \& E$ & $C V$ & Feulgen & $H \& E$ & $\mathrm{CV}$ & Feulgen \\
\hline 1. & 2 & 2 & 3 & 3 & 4.5 & 7 & 14 & 17 & 19 \\
\hline 2. & 0 & 1.5 & 3 & 4 & 7 & 7.5 & 8.5 & 8 & 10.5 \\
\hline 3. & 1 & 1.5 & 1 & 4 & 4 & 7.5 & 13 & 15 & 17.5 \\
\hline 4. & 0 & 0.5 & 1 & 4 & 4 & 6.5 & 11 & 14.5 & 19 \\
\hline 5. & 3 & 3.5 & 3 & 5 & 5 & 7 & 14 & 16 & 17 \\
\hline 6. & 2.5 & 3 & 5 & 8 & 9 & 9 & 13 & 19 & 20.5 \\
\hline 7. & 1 & 1 & 2 & 6 & 6.5 & 10 & 15 & 16 & 14 \\
\hline 8. & 0 & 0.5 & 1 & 5 & 8 & 8 & 15 & 14 & 18 \\
\hline 9. & 0 & 1 & 2 & 8 & 8 & 10.5 & 13 & 16 & 18.5 \\
\hline 10. & 1 & 2 & 1.5 & 6 & 7.5 & 8.5 & 14 & 15.5 & 16 \\
\hline
\end{tabular}

H\&E: Hematoxylin and eosin; CV: $1 \%$ crystal violet 


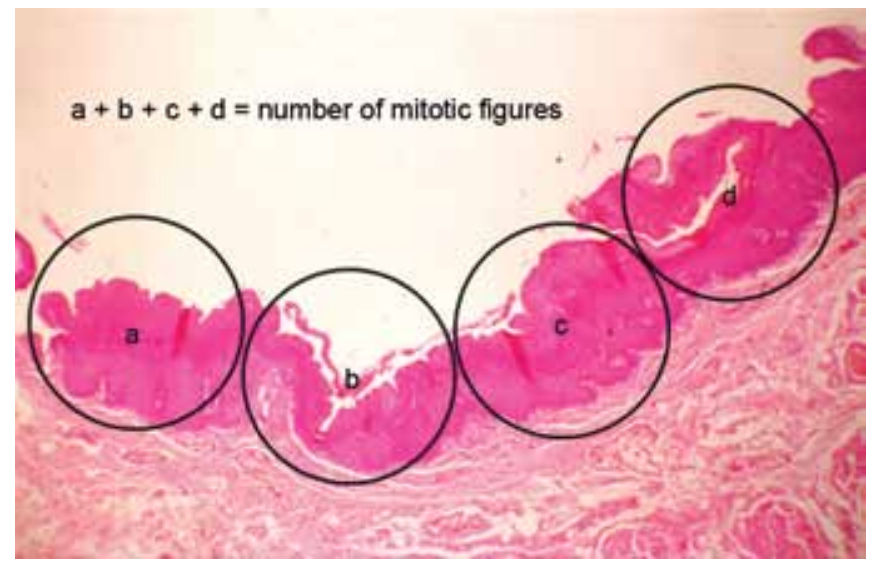

Fig. 1: Method of evaluation of MFs
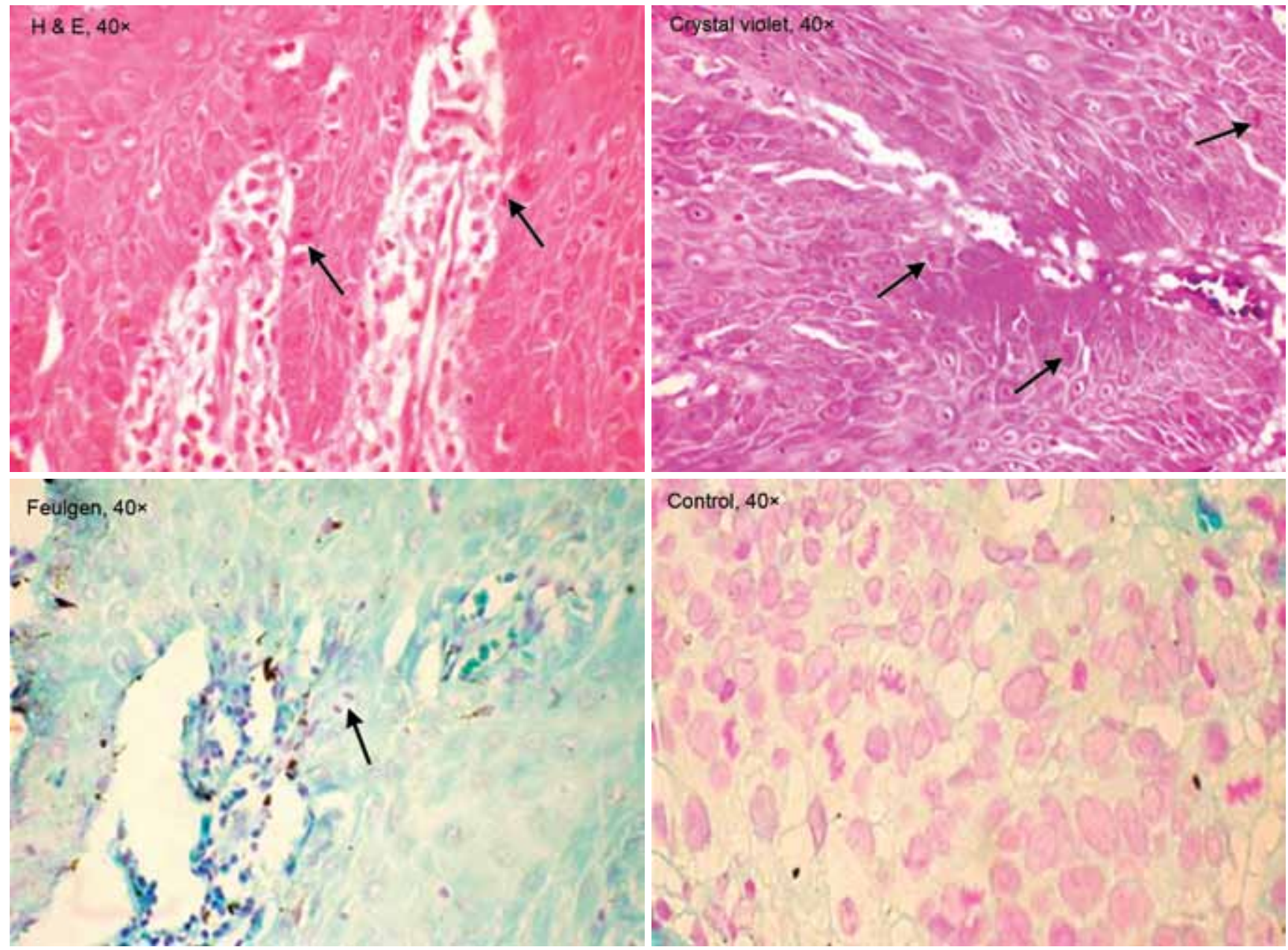

Fig. 2: H\&E, Feulgen and crystal violet stained sections showing MFs

and severe epithelial dysplasia was observed (Table 3 ). The $\mathrm{p}$-values between the various groups are shown in Table 2, and stained sections are shown in Figure 2.

Interobserver variability was calculated to be 0.98 which showed high agreement between the observers.

\section{DISCUSSIONS}

Mitosis generates new cells and is responsible for the growth and maintenance of an organism. It is divided into five stages: prophase, prometaphase, metaphase, anaphase and telophase, where each stage is characterized by a particular series of events ${ }^{1}$ (Fig. 3).

Carcinogenesis is the result of genetic alterations in nuclear DNA, which is evident as dysregulated mitosis and nucleoli numbers. Increased MFs and nucleoli counts are recognized cellular changes in oral epithelial dysplasia. Increased and abnormal mitosis hints at genetic damage, and has a significant role in carcinogenesis. Microscopic evaluation of mitotic activity is one of the most valuable prognostic indicators. 


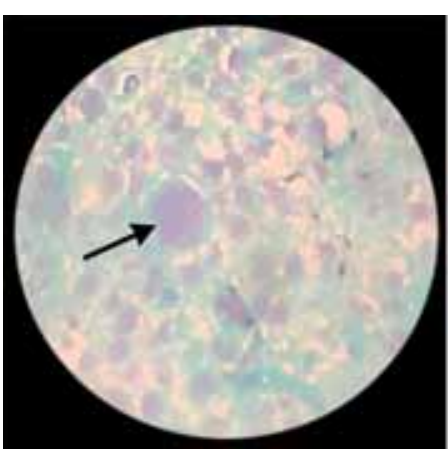

Prophase

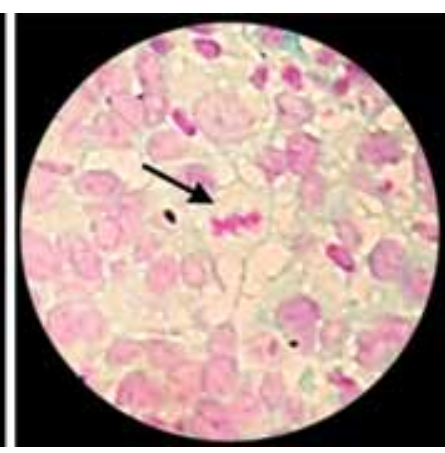

Metaphase

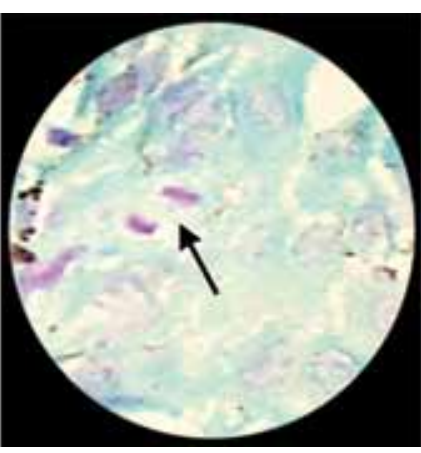

Anaphase

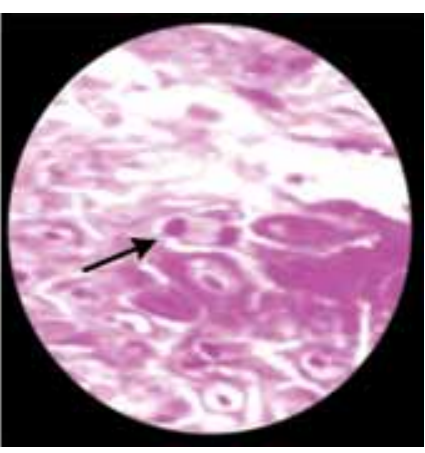

Telophase

Chromosomes cluster at opposite spindle poles, nuclear membrane assembles, and daughter cells are formed by cytokinesis ses to form compact MFs, and the metaphase plate nuclear membrane is lost

Fig. 3: Various stages of mitosis

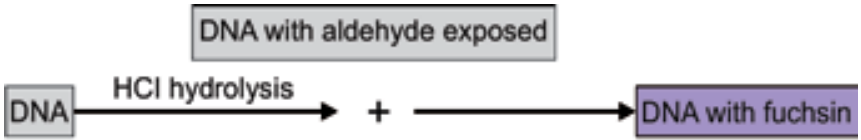

Leuco fuchsin

Fig. 4: Mechanism of action of Feulgen

The significance of MFs are:

1. Prognostication of a variety of neoplasms.

2. Mechanisms behind observed chromosomal aberration.

3. Assessing cellular proliferation and aid in histological grading.

Thus, easy identification and quantification of these figures is an indispensible aid in pathology.

New advanced techniques, like immunohistochemistry using Ki-67, PCNA and phosphohistone H3, autoradiographic determination of thymidine labeling to see for proliferation, flow cytometry, etc. are also available but their increased cost and time makes them difficult to use. A standardized, simple and quick stain with a set of welldefined criteria for the quantification of MFs will be effective and helpful. ${ }^{2,3}$

In the present study, we aimed to identify a simple, costeffective and rapid technique for identification of MFs and also to evaluate its role in histological grading of oral epithelial dysplasia. It was observed that Feulgen stain provided superior staining of MFs facilitating its identification as compared to $1 \%$ crystal violet and $\mathrm{H} \& \mathrm{E}$ stains. Distinguishing a MFs from pyknosis, apoptosis, karyorrhexis is important to prevent false positive results, and Feulgen stain provided excellent detail and morphology of a mitotic cell.

The method of Feulgen and Rossenbeck (1924) is a standard technique for demonstrating deoxyribose. Mild acid hydrolysis in $1 \mathrm{M} \mathrm{HCl}$ at $60^{\circ} \mathrm{C}$ is used to break the purine-deoxyribose bond and the resulting exposed aldehydes are then demonstrated by Schiff's reagent as depicted in Figure $4 .^{6}$
Comparison of the mitotic counts between various grades of oral epithelial dysplasia failed to yield any significant variations.

In a similar study conducted by Ankle MR to evaluate the selectivity of one percent crystal violet stain for MFs on normal oral mucosa, oral epithelial dysplasia and oral squamous cell carcinoma. One percent crystal violet staining was compared with routine $\mathrm{H} \& \mathrm{E}$ staining. Results showed statistically increased mean mitotic counts with $1 \%$ crystal violet stained sections of oral epithelial dysplasia and oral squamous cell carcinoma as compared to H\&E stained sections. ${ }^{2}$

In another study conducted by Jadhav KB, it was seen that $1 \%$ crystal violet provided a definite advantage over H\&E stained sections in selectively staining MFs. A significant increase in the number of MFs in crystal violet stained tissues was observed in oral epithelial dysplasia and oral squamous cell carcinoma. ${ }^{3}$

A study conducted by Division of Human Nutrition, Adelaide South Australia, to evaluate the selective stain for MFs in tissue sections of developing brain, large and small intestine, skin and liver from rat, sheep and guinea pigs. The staining was done with $1 \%$ crystal violet with nuclear fast red as counter satin. Results showed that tissue MFs were visible in low power and stood out clearly against red background. ${ }^{7}$

However, in the present study, we noted that Feulgen was superior in staining MFs as compared to $1 \%$ crystal violet. The purple-colored chromatin material against a green cytoplasm facilitated the distinct and highly selective identification of MFs even at low power. The use of Feulgen stain is reproducible, rapid, simple and feasible for the localization of MFs and can be routinely employed.

\section{CONCLUSION}

We strongly advocate the use of Feulgen stain for distinct and selective staining of MFs. Feulgen reaction is a reliable and specific histochemical method for nuclear DNA. When 
performed under carefully controlled conditions, it provides a reasonably good staining of nuclear DNA.

\section{REFERENCES}

1. Karp G. Cell and molecular biology: Concepts and experiments. 6th ed. USA: John Wiley and Sons; 2013. p. 560-604.

2. Ankle MR, Kale AD, Chandrimath S. Comparison of staining of mitotic figures by haematoxylin and eosin stained crystal violet stain in oral epithelial dysplasia and squamous cell carcinoma. Indian J Dent Res 2007;18(3):101-105.

3. Jadhav KB, Ahmed Mujib BR, Gupta N. Crystal violet stain as a selective stain for the assessment of mitotic figures in oral epithelial dysplasia and oral squamous cell carcinoma. Indian J Pathol Micobiol 2012;55(3):283-287.

4. Warnakulasuriya S, Reibel J, Bouquot J, Dabelsteen E. Oral epithelial dysplasia classification systems: predictive value, utility, weaknesses and scope for improvement. J Oral Pathol Med 2008;37:127-133.

5. Godkar PB, Godkar DP. Textbook of Medical Laboratory Technology. 2nd ed. Mumbai, India: Bhalani Publishing House; 2003. p. 1009-1011.

6. Bancroft JD. Theory of histological techniques. 6th ed. Philadelphia PA: Churchill Livingstone Elsevier; 2007.

7. Fraser FJ. A selective stain for mitotic figures particularly in the developing brain. Stain Technol 1982;57:219-223. 